\title{
Expanded Mixed Finite Element Method for the Two-Dimensional Sobolev Equation
}

\author{
Qing-li Zhao, ${ }^{1,2}$ Zong-cheng $\mathrm{Li}^{2}{ }^{2}$ and You-zheng Ding ${ }^{2}$ \\ ${ }^{1}$ School of Mathematics, Shandong University, Jinan 250100, China \\ ${ }^{2}$ School of Science, Shandong Jianzhu University, Jinan 250101, China \\ Correspondence should be addressed to Qing-li Zhao; zhaoqingliabc@163.com
}

Received 23 April 2013; Revised 7 June 2013; Accepted 7 June 2013

Academic Editor: Carlos Conca

Copyright (c) 2013 Qing-li Zhao et al. This is an open access article distributed under the Creative Commons Attribution License, which permits unrestricted use, distribution, and reproduction in any medium, provided the original work is properly cited.

Expanded mixed finite element method is introduced to approximate the two-dimensional Sobolev equation. This formulation expands the standard mixed formulation in the sense that three unknown variables are explicitly treated. Existence and uniqueness of the numerical solution are demonstrated. Optimal order error estimates for both the scalar and two vector functions are established.

\section{Introduction}

In this paper, we consider the following Sobolev equation:

$$
\begin{gathered}
u_{t}(\mathbf{x}, t)-\nabla \cdot\left\{a(\mathbf{x}, t) \nabla u_{t}+b_{1}(\mathbf{x}, t) \nabla u(\mathbf{x}, t)\right\} \\
=f(\mathbf{x}, t), \quad(\mathbf{x}, t) \in \Omega \times(0, T], \\
u(\mathbf{x}, t)=0, \quad(\mathbf{x}, t) \in \partial \Omega \times[0, T], \\
u(\mathbf{x}, 0)=u_{0}(\mathbf{x}), \quad \mathbf{x} \in \Omega,
\end{gathered}
$$

in a bounded domain $\Omega \subset R^{2}$ with Lipschitz continuous boundary $\partial \Omega$. Equation (1) has a wide range of applications in many mathematical and physical problems [1, 2], for example, the percolation theory when the fluid flows through the cracks, the transfer problem of the moisture in the soil, and the heat conduction problem in different materials. So there exists great and actual significance to research Sobolev equation. Up till now, there are some different schemes studied to solve this kind of equation (see [1-4] for instance).

The mixed finite element method, which is a finite element method [5] with constrained conditions, plays an important role in the research of the numerical solution for partial differential equations. Its general theory was proposed by Babuska [6] and Brezzi [7]. Falk and Osborn [8] improved their theory and expanded the adaptability of the mixed finite element method. The mixed finite element method (see $[9,10]$ for instance) is wildly used for the modeling of fluid flow and transport, as it provides accurate and locally mass conservative velocities.

The main motivation of the expanded mixed finite element method [11-15] is to introduce three (or more) auxiliary variables for practical problems, while the traditional finite element method and mixed finite element method can only approximate one and two variables, respectively. The expanded mixed finite element method also has some other advantages except introducing more variables. It can treat individual boundary conditions. Also, this method is suitable for differential equation with small coefficient (close to zero) which does not need to be inverted. Consequently, this method works for the problems with small diffusion or low permeability terms in fluid problems. Using this method, we can get optimal order error estimates for certain nonlinear problems, while standard mixed formulation sometimes gives only suboptimal error estimates.

The object of this paper is to present the expanded mixed finite element method for the Sobolev equation. We conduct theoretical analysis to study the existence and uniqueness and obtain optimal order error estimates. The rest of this paper is organized as follows. In Section 2, the mixed weak formulation and its mixed element approximation are considered. In Section 3, we prove the existence and uniqueness of approximation form. In Section 4, some lemmas are given. 
In Section 5, optimal order semidiscrete error estimates are established.

Throughout the paper, we will use $C$, with or without subscript, to denote a generic positive constant which does not depend on the discretization parameter $h$. Vectors will be expressed in boldface. At the same time, we show a useful $\varepsilon$ Cauchy inequality

$$
a b \leq \varepsilon a^{2}+\frac{1}{4 \varepsilon} b^{2}, \quad \varepsilon>0
$$

\section{Mixed Weak Form and Mixed Element Approximation}

For $1 \leq q \leq+\infty$ and $m$ any nonnegative integer, let

$$
W^{m, q}(\Omega)=\left\{f \in L^{q}(\Omega)\left|D^{\alpha} f \in L^{q}(\Omega),\right| \alpha \mid \leq m\right\}
$$

denote the Sobolev spaces [16] endowed with the norm

$$
\|f\|_{m, q, \Omega}=\left(\sum_{|\alpha| \leq m}\left\|D^{\alpha} f\right\|_{L^{q}(\Omega)}^{q}\right)^{1 / q}
$$

(the subscript $\Omega$ will always be omitted).

Let $H^{m}(\Omega)=W^{m, 2}(\Omega)$ with the norm $\|\cdot\|_{m}=\|\cdot\|_{m, 2}$. The notation $\|\cdot\|$ will mean $\|\cdot\|_{L^{2}(\Omega)}$ or $\|\cdot\|_{L^{2}(\Omega)^{2}}$. We denote by $\left(\right.$, ) the inner product in either $L^{2}(\Omega)$ or $L^{2}(\Omega)^{2}$; that is,

$$
(\xi, \eta)=\int_{\Omega} \xi \eta d \mathbf{x}, \quad(\xi, \boldsymbol{\eta})=\int_{\Omega} \xi \cdot \boldsymbol{\eta} d \mathbf{x}
$$

The notation $\langle$,$\rangle denotes the L^{2}$-inner product on the boundary of $\Omega$

$$
\langle\xi, \eta\rangle=\int_{\partial \Omega} \xi \eta d s .
$$

To formulate the weak form, let $b=b_{1} / a$; we introduce two vector variables

$$
\lambda=-\nabla u_{t}-b \nabla u, \quad \sigma=a \lambda=-a \nabla u_{t}-b_{1} \nabla u
$$

Letting $\mathbf{c}=-\nabla b$, we rewrite (1) as the following system:

$$
\begin{gathered}
u_{t}+\nabla \cdot \boldsymbol{\sigma}=f, \quad(\mathbf{x}, t) \in \Omega \times(0, T], \\
\lambda+\nabla u_{t}-\nabla(b u)+\mathbf{c} u=0, \quad(\mathbf{x}, t) \in \Omega \times(0, T], \\
\boldsymbol{\sigma}-a \boldsymbol{\lambda}=0, \quad(\mathbf{x}, t) \in \Omega \times(0, T], \\
u(\mathbf{x}, t)=0, \quad(\mathbf{x}, t) \in \partial \Omega \times(0, T], \\
u(\mathbf{x}, 0)=u_{0}(\mathbf{x}), \quad \mathbf{x} \in \Omega .
\end{gathered}
$$

Let $\mathbf{n}$ be the unit exterior normal vector to the boundary of $\Omega$. Then (8) is formulated in the following expanded mixed weak form: find $(u, \lambda, \sigma) \in W \times \boldsymbol{\Lambda} \times \mathbf{V}$, such that

$$
\begin{gathered}
\left(u_{t}, w\right)+(\operatorname{div} \boldsymbol{\sigma}, w)=(f, w), \\
\forall w \in W, 0<t \leq T, \\
(\boldsymbol{\lambda}, \mathbf{v})-\left(u_{t}+b u, \operatorname{div} \mathbf{v}\right)+(\mathbf{c} u, \mathbf{v})=0, \\
\forall \mathbf{v} \in \mathbf{V}, 0<t \leq T, \\
(\boldsymbol{\sigma}, \boldsymbol{\tau})-(a \boldsymbol{\lambda}, \boldsymbol{\tau})=0, \quad \forall \boldsymbol{\tau} \in \boldsymbol{\Lambda}, 0<t \leq T, \\
(u(0), w)=\left(u_{0}, w\right), \quad \forall w \in W,
\end{gathered}
$$

where

$$
\begin{gathered}
W=L^{2}(\Omega), \quad \Lambda=L^{2}(\Omega)^{2} \\
\mathbf{V}=H(\operatorname{div}, \Omega)=\left\{\mathbf{v} \in L^{2}(\Omega)^{2} \mid \nabla \cdot \mathbf{v} \in L^{2}(\Omega)\right\} .
\end{gathered}
$$

Let $T_{h}$ be a quasiregular polygonalization of $\Omega$ (by triangles or rectangles), with $h$ being the maximum diameter of the elements of the polygonalization. Let $W_{h} \times \Lambda_{h} \times \mathbf{V}_{h}$ be a conforming mixed element space with index $k$ and discretization parameter $h$. $W_{h} \times \boldsymbol{\Lambda}_{h} \times \mathbf{V}_{h}$ is an approximation to $W \times \Lambda \times \mathbf{V}$. There are many conforming (or compatible) mixed element function spaces such as Raviart-Thomas elements [17], BDFM elements [18, 19]. Some RT type mixed elements are listed in Table 1 . Here, $P_{k}(T)$ is the polynomial up to $k$ order in two-dimensional domain used in triangle, while $Q_{k, l}(T)$ is the polynomial up to $k, l$ in each dimension used in rectangle.

Replacing the three variables by their approximation, we get the expanded mixed finite element approximation problem: find $\left(u_{h}, \lambda_{h}, \sigma_{h}\right) \in W_{h} \times \Lambda_{h} \times \mathbf{V}_{h}$, such that

$$
\begin{gathered}
\left(u_{h, t}, w\right)+\left(\operatorname{div} \boldsymbol{\sigma}_{h}, w\right)=(f, w), \\
\forall w \in W_{h}, 0<t \leq T, \\
\left(\boldsymbol{\lambda}_{h}, \mathbf{v}\right)-\left(u_{h, t}+b u_{h}, \operatorname{div} \mathbf{v}\right)+\left(\mathbf{c} u_{h}, \mathbf{v}\right)=0, \\
\forall \mathbf{v} \in \mathbf{V}_{h}, 0<t \leq T, \\
\left(\boldsymbol{\sigma}_{h}, \boldsymbol{\tau}\right)-\left(a \boldsymbol{\lambda}_{h}, \boldsymbol{\tau}\right)=0, \quad \forall \boldsymbol{\tau} \in \boldsymbol{\Lambda}_{h}, 0<t \leq T, \\
(u(0), w)=\left(\tilde{u}_{0}, w\right), \quad \forall w \in W_{h},
\end{gathered}
$$

where

$$
\begin{aligned}
& W_{h}(E)=\left\{\omega \in W:\left.\omega\right|_{E} \in W_{h}(E), \forall E \in T_{h}\right\}, \\
& \boldsymbol{\Lambda}_{h}(E)=\left\{\boldsymbol{\tau} \in \boldsymbol{\Lambda}:\left.\boldsymbol{\tau}\right|_{E} \in \mathbf{V}_{h}(E), \forall E \in T_{h}\right\}, \\
& \mathbf{V}_{h}(E)=\left\{\mathbf{v} \in \mathbf{V}:\left.\mathbf{v}\right|_{E} \in \mathbf{V}_{h}(E), \forall E \in T_{h}\right\} .
\end{aligned}
$$

The error analysis next makes use of three projection operators. The first operator is the Raviart-Thomas projection (or Brezzi-Douglas-Marini projection) $\Pi_{h}: \mathbf{V} \rightarrow \mathbf{V}_{h} ; \Pi_{h}$ satisfies

$$
\left(\nabla \cdot\left(\mathbf{v}-\Pi_{h} \mathbf{v}\right), \omega_{h}\right)=0, \quad \forall \omega_{h} \in W_{h} .
$$


TABLE 1: Some RT type mixed elements.

\begin{tabular}{lccc}
\hline Dim & Element & $\mathbf{V}_{h}(E)$ & $W_{h}(E)$ \\
\hline 2D & Triangle & $\mathrm{RT}_{k}(T)=P_{k}(E)^{2} \oplus \mathbf{x} P_{k}(E)$ & $P_{k}(E)$ \\
2D & Rectangle & $\mathrm{RT}_{[k]}(T)=Q_{k+1, k}(E) \oplus Q_{k, k+1}(E)$ & $Q_{k, k}(E)$ \\
\hline
\end{tabular}

The following approximation properties are well known.

$$
\begin{gathered}
\left\|\mathbf{v}-\Pi_{h} \mathbf{v}\right\| \leq C h^{r}\|\mathbf{v}\|_{r}, \quad \frac{1}{2} \leq r \leq k+1, \\
\left\|\nabla \cdot\left(\mathbf{v}-\Pi_{h} \mathbf{v}\right)\right\|_{r} \leq C h^{r}\|\nabla \cdot \mathbf{v}\|_{r}, \quad 0 \leq r \leq k+1 .
\end{gathered}
$$

The other two operators are the standard $L^{2}$-projection [5] $P_{h}$ and $R_{h}$ onto $W_{h}$ and $\boldsymbol{\Lambda}_{h}$, respectively,

$$
\begin{gathered}
\left(\omega-P_{h} \omega, \nabla \cdot \mathbf{v}_{h}\right)=0, \quad \forall \omega \in W, \mathbf{v}_{h} \in \mathbf{V}_{h}, \\
\left(\boldsymbol{\tau}-R_{h} \boldsymbol{\tau}, \boldsymbol{\tau}_{h}\right)=0, \quad \forall \boldsymbol{\tau} \in \boldsymbol{\Lambda}, \boldsymbol{\tau}_{h} \in \boldsymbol{\Lambda}_{h} .
\end{gathered}
$$

They have the approximation properties

$$
\begin{gathered}
\left\|\omega-P_{h} \omega\right\| \leq C h^{r}\|\omega\|_{r}, \quad 0 \leq r \leq k+1, \\
\left\|\boldsymbol{\tau}-R_{h} \tau\right\| \leq C h^{r}\|\boldsymbol{\mu}\|_{r}, \quad 0 \leq r \leq k+1 .
\end{gathered}
$$

The two projections $\Pi_{h}$ and $P_{h}$ preserve the commuting property

$$
\operatorname{div} \circ \Pi_{h}=P_{h} \circ \operatorname{div}: H^{1}(\Omega)^{2} \longrightarrow W_{h} .
$$

\section{Existence and Uniqueness of Approximation Form}

In this section, we consider the existence and uniqueness of the solution of (11).

\section{Lemma 1. Equation (11) has a unique solution.}

Proof. In fact, this equation is linear; it suffices to show that the associated homogeneous system

$$
\begin{gathered}
\left(u_{h, t}, w\right)+\left(\operatorname{div} \boldsymbol{\sigma}_{h}, w\right)=0, \\
\forall w \in W_{h}, 0<t \leq T, \\
\left(\lambda_{h}, \mathbf{v}\right)-\left(u_{h, t}+b u_{h}, \operatorname{div} \mathbf{v}\right)+\left(\mathbf{c} u_{h}, \mathbf{v}\right)=0, \\
\forall \mathbf{v} \in \mathbf{V}_{h}, 0<t \leq T, \\
\left(\boldsymbol{\sigma}_{h}, \boldsymbol{\tau}\right)-\left(a \boldsymbol{\lambda}_{h}, \boldsymbol{\tau}\right)=0, \\
\forall \boldsymbol{\tau} \in \boldsymbol{\Lambda}_{h}, 0<t \leq T, \\
(u(0), w)=0, \quad \forall w \in W_{h},
\end{gathered}
$$

has only the trivial solution. In the first equation of (19), if we take $\omega=u_{h, t}$ and $\omega=\operatorname{div} \sigma_{h}$, respectively, then we have that

$$
\begin{aligned}
& \left\|u_{h, t}\right\| \leq\left\|\operatorname{div} \sigma_{h}\right\|, \\
& \left\|\operatorname{div} \sigma_{h}\right\| \leq\left\|u_{h, t}\right\| .
\end{aligned}
$$

By (20), it is easy to see that

$$
\left\|\operatorname{div} \sigma_{h}\right\|=\left\|u_{h, t}\right\| .
$$

Choosing $\omega=\operatorname{div} \boldsymbol{\sigma}_{h}, \mathbf{v}=\boldsymbol{\sigma}_{h}$, and $\boldsymbol{\tau}=\boldsymbol{\lambda}_{h}$ in (19), we get

$$
\left(a \lambda_{h}, \lambda_{h}\right)+\left(\operatorname{div} \sigma_{h}, \operatorname{div} \sigma_{h}\right)=\left(b u_{h}, \operatorname{div} \sigma_{h}\right)-\left(c u_{h}, \sigma_{h}\right) .
$$

In the third equation of (19), letting $\tau=\sigma_{h}$ and $\tau=\lambda_{h}$, respectively, then

$$
\begin{gathered}
\left\|\boldsymbol{\sigma}_{h}\right\| \leq C\left\|\boldsymbol{\lambda}_{h}\right\|, \\
\left\|\lambda_{h}\right\| \leq C\left\|\boldsymbol{\sigma}_{h}\right\| .
\end{gathered}
$$

Using the $\varepsilon$-Cauchy inequality to (22), we have

$$
a_{0}\left\|\lambda_{h}\right\|^{2}+\left\|\operatorname{div} \sigma_{h}\right\|^{2} \leq C\left\|u_{h}\right\|^{2}+\frac{1}{2}\left\|\operatorname{div} \sigma_{h}\right\|^{2}+\frac{a_{0}}{2}\left\|\lambda_{h}\right\|^{2} .
$$

Note that (21), we deserve

$$
\begin{gathered}
\left\|\lambda_{h}\right\| \leq C\left\|u_{h}\right\|, \\
\left\|u_{h, t}\right\| \leq C\left\|u_{h}\right\| .
\end{gathered}
$$

From (27), we know

$$
\left\|u_{h}\right\|=\left\|\int_{0}^{t} u_{h, t} d t\right\| \leq C \int_{0}^{t}\left\|u_{h, t}\right\| d t \leq C \int_{0}^{t}\left\|u_{h}\right\| d t .
$$

Using Gronwall's inequality to (28), we can prove $u_{h}=0$. Further, from (26) and (23), we know that $\boldsymbol{\lambda}_{h}=\mathbf{0}, \boldsymbol{\sigma}_{h}=\mathbf{0}$. The proof is completed.

\section{Some Lemmas}

In the study of parabolic equations, we usually introduce a mixed elliptic projection associated with our equations. Define a map: find $\left(\widetilde{u}_{h}, \widetilde{\lambda}_{h}, \widetilde{\boldsymbol{\sigma}}_{h}\right) \in W_{h} \times \boldsymbol{\Lambda}_{h} \times \mathbf{V}_{h}$, such that

$$
\begin{gathered}
\left(\operatorname{div}\left(\boldsymbol{\sigma}-\widetilde{\boldsymbol{\sigma}}_{h}\right), w\right)=0, \quad \forall w \in W_{h}, 0<t \leq T, \\
\left(\boldsymbol{\lambda}-\widetilde{\boldsymbol{\lambda}}_{h}, \mathbf{v}\right)-\left(u_{t}-\widetilde{u}_{h, t}+b\left(u-\widetilde{u}_{h}\right), \operatorname{div} \mathbf{v}\right)+\left(\mathbf{c}\left(u-\widetilde{u}_{h}\right), \mathbf{v}\right) \\
=0, \quad \forall \mathbf{v} \in \mathbf{V}_{h}, 0<t \leq T, \\
\left(\sigma-\widetilde{\boldsymbol{\sigma}}_{h}, \boldsymbol{\tau}\right)-\left(a\left(\lambda-\widetilde{\lambda}_{h}\right), \boldsymbol{\tau}\right)=0, \quad \forall \boldsymbol{\tau} \in \boldsymbol{\Lambda}_{h}, \quad 0<t \leq T, \\
\left(u(0)-\widetilde{u}_{h}(0), w\right)=0, \quad \forall w \in W_{h} .
\end{gathered}
$$

Similarly to Lemma 1, we can prove that system (29) has a unique solution.

Now we give some error estimates of $\left(\widetilde{u}_{h}, \widetilde{\lambda}_{h}, \widetilde{\sigma}_{h}\right)$. Define

$$
\begin{gathered}
u-\widetilde{u}_{h}=\left(p_{h} u-\widetilde{u}_{h}\right)+\left(u-p_{h} u\right)=u_{1}+u_{2}, \\
\lambda_{1}=\lambda-\tilde{\lambda}_{h}, \quad \sigma_{1}=\sigma-\widetilde{\sigma}_{h} .
\end{gathered}
$$


System (29) can be rewritten as follows:

$$
\begin{gathered}
\left(\operatorname{div} \boldsymbol{\sigma}_{1}, w\right)=0, \quad \forall w \in W_{h}, \\
\left(\lambda_{1}, \mathbf{v}\right)-\left(u_{1, t}+b\left(u_{1}+u_{2}\right), \operatorname{div} \mathbf{v}\right)+\left(\mathbf{c}\left(u_{1}+u_{2}\right), \mathbf{v}\right)=0 \\
\forall \mathbf{v} \in \mathbf{V}_{h}, \\
\left(\boldsymbol{\sigma}_{1}, \boldsymbol{\tau}\right)-\left(a \lambda_{1}, \boldsymbol{\tau}\right)=0, \quad \forall \boldsymbol{\tau} \in \Lambda_{h} . \\
\left(u(0)-\tilde{u}_{h}(0), w\right)=0, \quad \forall w \in W_{h} .
\end{gathered}
$$

Now we consider the estimates of $u_{1}$ and $u_{1, t}$.

Lemma 2. Let $\left(u_{1}, \lambda_{1}, \sigma_{1}\right)$ be the solution of system (31). If $u, \lambda$, and $\sigma$ are sufficiently smooth, then there exist positive constants $C$ such that

$$
\begin{gathered}
\left\|u_{1}\right\| \leq C \int_{0}^{t}\left(h\left\|\lambda_{1}\right\|+h^{1-\delta_{k 0}}\left\|\lambda_{1}\right\|+h\left\|u_{2}\right\|+\left\|u_{2}\right\|_{-1}\right) d t, \\
\left\|u_{1, t}\right\| \leq C\left(h\left\|\lambda_{1}\right\|+h^{1-\delta_{k 0}}\left\|\lambda_{1}\right\|+h\left\|u_{2}\right\|+\left\|u_{2}\right\|_{-1}+\left\|u_{1}\right\|\right),
\end{gathered}
$$

where $\delta_{k 0}=0$ for $k=0$, and $\delta_{k 0}=0$ for $k \geq 1$.

Proof. Let $\phi \in H^{2}(\Omega) \bigcap H_{0}^{1}(\Omega)$ be the solution of the following problem:

$$
\begin{gathered}
\nabla \cdot(a \nabla \phi)=\psi, \quad \forall x \in \Omega, \\
\left.\phi\right|_{\partial \Omega}=0 .
\end{gathered}
$$

Then we know

$$
\|\phi\|_{2} \leq C\|\psi\|
$$

For $0<t \leq T$, from the second equation of (31), we have that

$$
\begin{aligned}
\left(u_{1, t}, \psi\right)= & \left(u_{1, t}, \operatorname{div}(a \nabla \phi)\right) \\
= & \left(u_{1, t}, \operatorname{div} \Pi_{h}(a \nabla \phi)\right) \\
= & \left(\lambda_{1}, \Pi_{h}(a \nabla \phi)\right)+\left(\mathbf{c}\left(u_{1}+u_{2}\right), \Pi_{h}(a \nabla \phi)\right) \\
& -\left(b\left(u_{1}+u_{2}\right), \operatorname{div} \Pi_{h}(a \nabla \phi)\right) \\
= & I_{1}+I_{2}+I_{3} .
\end{aligned}
$$

It is easy to see that

$$
\begin{aligned}
I_{1} & =\left(\lambda_{1}, \Pi_{h}(a \nabla \phi)\right) \\
& =\left(\lambda_{1}, \Pi_{h}(a \nabla \phi)-a \nabla \phi\right)+\left(\lambda_{1}, a \nabla \phi\right) \\
& =\left(\lambda_{1}, \Pi_{h}(a \nabla \phi)-a \nabla \phi\right)+\left(a \lambda_{1}, \nabla \phi\right) \\
& =E_{1}+E_{2},
\end{aligned}
$$$$
E_{1} \leq C h\left\|\lambda_{1}\right\|\|\phi\|_{2} \leq C h\left\|\lambda_{1}\right\|\|\psi\|,
$$$$
E_{2}=\left(a \lambda_{1}, \nabla\left(\phi-p_{h} \phi\right)\right)+\left(a \lambda_{1}, \nabla\left(p_{h} \phi\right)\right)
$$$$
=\left(a \lambda_{1}, \nabla\left(\phi-p_{h} \phi\right)\right)+\left(\sigma_{1}, \nabla\left(p_{h} \phi\right)\right)
$$$$
=\left(a \lambda_{1}, \nabla\left(\phi-p_{h} \phi\right)\right)-\left(\operatorname{div} \sigma_{1},\left(p_{h} \phi\right)\right)
$$$$
=\left(a \lambda_{1}, \nabla\left(\phi-p_{h} \phi\right)\right)
$$$$
\leq C\left\|\lambda_{1}\right\|\left\|\nabla\left(\phi-p_{h} \phi\right)\right\|
$$$$
\leq C h^{1-\delta_{k o}}\|\phi\|_{2}\left\|\lambda_{1}\right\|
$$$$
\leq C h^{1-\delta_{k o}}\|\psi\|\left\|\lambda_{1}\right\|
$$

Now, we estimate $I_{2}$,

$$
\begin{aligned}
\left(\mathbf{c} u_{1},\right. & \left.\Pi_{h}(a \nabla \phi)\right) \\
\leq & C\left\|u_{1}\right\|\left\|\Pi_{h}(a \nabla \phi)-a \nabla \phi+a \nabla \phi\right\| \\
\leq & C\left\|u_{1}\right\|(h\|\nabla \phi\|+\|\nabla \phi\|) \\
\leq & C\left\|u_{1}\right\|\|\psi\|, \\
\left(\mathbf{c} u_{2},\right. & \left.\Pi_{h}(a \nabla \phi)\right) \\
& =\left(\mathbf{c} u_{2}, \Pi_{h}(a \nabla \phi)-a \nabla \phi\right)+\left(\mathbf{c} u_{2}, a \nabla \phi\right) \\
\leq & C\left(h\left\|u_{2}\right\|+\left\|u_{2}\right\|_{-1}\right)\|\psi\| .
\end{aligned}
$$

We turn to consider $I_{3}$,

$$
\begin{gathered}
\left(-b u_{1}, \operatorname{div} \Pi_{h}(a \nabla \phi)\right) \\
\leq C\left\|u_{1}\right\|\|\nabla \phi\| \leq C\left\|u_{1}\right\|\|\psi\|, \\
\left(-b u_{2}, \operatorname{div} \Pi_{h}(a \nabla \phi)\right) \\
\leq-\left(\left(b-p_{h}^{0} b\right) u_{2}, \operatorname{div} \Pi_{h}(a \nabla \phi)\right) \\
\quad-\sum_{e \in T_{h}}\left(\left(p_{h}^{0} b\right) u_{2}, \operatorname{div} \Pi_{h}(a \nabla \phi)\right)_{e} \\
=-\left(\left(b-p_{h}^{0} b\right) u_{2}, \operatorname{div} \Pi_{h}(a \nabla \phi)\right) \\
\leq C h\left\|u_{2}\right\|\|\psi\|,
\end{gathered}
$$

where $p_{h}^{0} b$ is the piecewise constant interpolation of function $b$. Using the previous estimates, we obtain

$\left\|u_{1, t}\right\|$

$$
\leq C\left(h\left\|\lambda_{1}\right\|+h^{1-\delta_{k 0}}\left\|\lambda_{1}\right\|+h\left\|u_{2}\right\|+\left\|u_{2}\right\|_{-1}+\left\|u_{1}\right\|\right) .
$$


So we deserve

$$
\begin{aligned}
&\left\|u_{1}\right\|=\left\|\int_{0}^{t} u_{1, t} d t\right\| \\
& \leq C \int_{0}^{t}\left\|u_{1, t}\right\| d t \\
& \leq C \int_{0}^{t}\left(h\left\|\lambda_{1}\right\|+h^{1-\delta_{k 0}}\left\|\lambda_{1}\right\|+h\left\|u_{2}\right\|\right. \\
&\left.+\left\|u_{2}\right\|_{-1}+\left\|u_{1}\right\|\right) d t .
\end{aligned}
$$

Applying Gronwall's inequality to (40), we have

$$
\begin{aligned}
& \left\|u_{1}\right\| \\
& \quad \leq C \int_{0}^{t}\left(h\left\|\lambda_{1}\right\|+h^{1-\delta_{k 0}}\left\|\lambda_{1}\right\|+h\left\|u_{2}\right\|+\left\|u_{2}\right\|_{-1}\right) d t .
\end{aligned}
$$

Noting the estimates of $I_{1}, I_{2}$, and $I_{3}$, the proof is completed.

Define

$$
\begin{gathered}
\|u, \lambda, \boldsymbol{\sigma}\|_{r}=\|u\|_{r}+\|\lambda\|_{r}+\|\boldsymbol{\sigma}\|_{r}, \\
\mid\|u, \lambda, \boldsymbol{\sigma}\| \|_{r}=\int_{0}^{t}\left(\|u\|_{r}+\|\lambda\|_{r}+\|\boldsymbol{\sigma}\|_{r}\right) d t .
\end{gathered}
$$

Lemma 3. Let $(u, \lambda, \sigma)$ and $\left(\widetilde{u}_{h}, \widetilde{\lambda}_{h}, \widetilde{\boldsymbol{\sigma}}_{h}\right)$ be the solution of (19) and (29), respectively. If $u, \lambda$, and $\sigma$ are sufficiently smooth, then there exist positive constants $C$ such that

(I) if $k \geq 0$, then

$$
\begin{gathered}
\left\|\operatorname{div}\left(\boldsymbol{\sigma}-\widetilde{\boldsymbol{\sigma}}_{h}\right)\right\| \leq C h^{r}\|\boldsymbol{\sigma}\|_{r+1}, \quad 0 \leq r \leq k+1, \\
\left\|p_{h} u-\widetilde{u}_{h}\right\| \leq\left. C h^{r+1-\delta_{k 0}}\|u, \lambda, \boldsymbol{\sigma}\|\right|_{r}, \quad 1 \leq r \leq k+1, \\
\left\|p_{h} u_{t}-\widetilde{u}_{h, t}\right\| \leq C h^{r+1-\delta_{k 0}}\|u, \lambda, \boldsymbol{\sigma}\|_{r}, \quad 1 \leq r \leq k+1,
\end{gathered}
$$

(II) if $k=0$, then

$$
\begin{aligned}
& \left\|u-\widetilde{u}_{h}\right\| \leq C h\left(\|u\|_{1}+|\|u, \lambda, \sigma\||_{1}\right), \\
& \left\|u_{t}-\widetilde{u}_{h, t}\right\| \leq C h\left(\left\|u_{t}\right\|_{1}+\|u, \lambda, \sigma\|_{1}+|\|u, \lambda, \sigma\||_{1}\right) \text {, } \\
& \left\|\lambda-\tilde{\lambda}_{h}\right\| \leq C h\left(\|u, \lambda, \sigma\|_{1}+\left.\|u, \lambda, \sigma\|\right|_{1}\right), \\
& \left\|\boldsymbol{\sigma}-\widetilde{\boldsymbol{\sigma}}_{h}\right\| \leq \operatorname{Ch}\left(\|u, \lambda, \boldsymbol{\sigma}\|_{1}+\left.\|u, \lambda, \boldsymbol{\sigma}\|\right|_{1}\right),
\end{aligned}
$$

(III) if $k \geq 1,2 \leq r \leq k+1$, then

$$
\begin{gathered}
\left\|u-\widetilde{u}_{h}\right\| \leq C h^{r}\left(\|u\|_{r}+\mid\|u, \lambda, \boldsymbol{\sigma}\|_{r-1}\right) \\
\left\|u_{t}-\widetilde{u}_{h, t}\right\| \leq C h^{r}\left(\left\|u_{t}\right\|_{r}+\|u, \lambda, \boldsymbol{\sigma}\|_{r}+\mid\|u, \lambda, \boldsymbol{\sigma}\|_{r-1}\right), \\
\left\|\lambda-\widetilde{\lambda}_{h}\right\| \leq C h^{r}\left(\|u, \lambda, \sigma\|_{r}+\|u, \lambda, \boldsymbol{\sigma}\|_{r-1}\right) \\
\left\|\boldsymbol{\sigma}-\widetilde{\boldsymbol{\sigma}}_{h}\right\| \leq C h^{r}\left(\|u, \lambda, \boldsymbol{\sigma}\|_{r}+\mid\|u, \lambda, \boldsymbol{\sigma}\|_{r-1}\right)
\end{gathered}
$$

Proof. For $0<t \leq T$, the proof proceeds in three steps as follows. know

(I) In the first equation of (29), taking $\omega=\operatorname{div} \sigma_{1}$, we

$$
\begin{aligned}
& \left(\operatorname{div} \sigma_{1}, \operatorname{div} \sigma_{1}\right) \\
& \quad=\left(\operatorname{div} \sigma_{1}, \operatorname{div}\left(\boldsymbol{\sigma}-\Pi_{h} \boldsymbol{\sigma}\right)\right) \\
& \quad \leq\left\|\operatorname{div} \sigma_{1}\right\|\left\|\operatorname{div}\left(\boldsymbol{\sigma}-\Pi_{h} \boldsymbol{\sigma}\right)\right\|,
\end{aligned}
$$

which implies that

$$
\begin{aligned}
\left\|\operatorname{div} \sigma_{1}\right\| & \leq\left\|\operatorname{div}\left(\boldsymbol{\sigma}-\Pi_{h} \boldsymbol{\sigma}\right)\right\| \\
& \leq C h^{r}\|\boldsymbol{\sigma}\|_{r+1}, \quad 0 \leq r \leq k+1 .
\end{aligned}
$$

Choosing $\omega \in W_{h}$, note that $\left(\operatorname{div}\left(\boldsymbol{\sigma}-\widetilde{\boldsymbol{\sigma}}_{h}\right), \omega\right)=0$ and $(\operatorname{div}(\boldsymbol{\sigma}-$ $\left.\left.\Pi_{h} \boldsymbol{\sigma}\right), \omega\right)=0$, we get

$$
\operatorname{div}\left(\Pi_{h} \boldsymbol{\sigma}-\widetilde{\boldsymbol{\sigma}}_{h}\right)=0 .
$$

Combing (15) with (56), we can prove (43).

(II) In (29), letting $\mathbf{v}=\Pi_{h} \boldsymbol{\sigma}-\widetilde{\boldsymbol{\sigma}}_{h}$, together with (43), we obtain

$$
\left(\boldsymbol{\lambda}-\widetilde{\boldsymbol{\lambda}}_{h}, \Pi_{h} \boldsymbol{\sigma}-\widetilde{\boldsymbol{\sigma}}_{h}\right)-\left(\mathbf{c}\left(u-\widetilde{u}_{h}\right), \Pi_{h} \boldsymbol{\sigma}-\widetilde{\boldsymbol{\sigma}}_{h}\right)=0 .
$$

Hence

$$
\left(R_{h} \lambda-\tilde{\boldsymbol{\lambda}}_{h}, \Pi_{h} \boldsymbol{\sigma}-\widetilde{\boldsymbol{\sigma}}_{h}\right)-\left(\mathbf{c}\left(u-\widetilde{u}_{h}\right), \Pi_{h} \boldsymbol{\sigma}-\widetilde{\boldsymbol{\sigma}}_{h}\right)=0 .
$$

In the third equation of (29), choosing $\tau=R_{h} \lambda-\widetilde{\lambda}_{h}$, we get

$$
\left(\boldsymbol{\sigma}-\tilde{\boldsymbol{\sigma}}_{h}, R_{h} \boldsymbol{\lambda}-\tilde{\boldsymbol{\lambda}}_{h}\right)=\left(a\left(\boldsymbol{\lambda}-\tilde{\boldsymbol{\lambda}}_{h}\right), R_{h} \boldsymbol{\lambda}-\tilde{\boldsymbol{\lambda}}_{h}\right) .
$$

So we know

$$
\begin{aligned}
(\boldsymbol{\sigma}- & \left.\Pi_{h} \boldsymbol{\sigma}, R_{h} \boldsymbol{\lambda}-\widetilde{\boldsymbol{\lambda}}_{h}\right)+\left(\Pi_{h} \boldsymbol{\sigma}-\widetilde{\boldsymbol{\sigma}}_{h}, R_{h} \boldsymbol{\lambda}-\widetilde{\boldsymbol{\lambda}}_{h}\right) \\
= & \left(a\left(\boldsymbol{\lambda}-\widetilde{\boldsymbol{\lambda}}_{h}\right), R_{h} \boldsymbol{\lambda}-\widetilde{\boldsymbol{\lambda}}_{h}\right) .
\end{aligned}
$$

Note that (44) and (45), we obtain

$$
\begin{aligned}
\left(\Pi_{h} \boldsymbol{\sigma}\right. & \left.-\widetilde{\boldsymbol{\sigma}}_{h}, R_{h} \boldsymbol{\lambda}-\widetilde{\boldsymbol{\lambda}}_{h}\right) \\
& =\left(\mathbf{c}\left(u_{h}-\widetilde{u}_{h}\right), \Pi_{h} \boldsymbol{\sigma}-\widetilde{\boldsymbol{\sigma}}_{h}\right) \\
& =\left(a\left(\boldsymbol{\lambda}-\widetilde{\boldsymbol{\lambda}}_{h}\right), R_{h} \boldsymbol{\lambda}-\widetilde{\boldsymbol{\lambda}}_{h}\right)-\left(\boldsymbol{\sigma}-\Pi_{h} \boldsymbol{\sigma}, R_{h} \boldsymbol{\lambda}-\widetilde{\boldsymbol{\lambda}}_{h}\right),
\end{aligned}
$$

which implies that

$$
\begin{aligned}
\left(\Pi_{h} \boldsymbol{\sigma}\right. & \left.-\widetilde{\boldsymbol{\sigma}}_{h}, R_{h} \boldsymbol{\lambda}-\tilde{\boldsymbol{\lambda}}_{h}\right) \\
& \leq C\left\|u-\widetilde{u}_{h}\right\|\left\|\Pi_{h} \boldsymbol{\sigma}-\widetilde{\boldsymbol{\sigma}}_{h}\right\| \\
& \leq C\left(\left\|u_{1}\right\|+\left\|u_{2}\right\|\right)\left\|\Pi_{h} \boldsymbol{\sigma}-\widetilde{\boldsymbol{\sigma}}_{h}\right\| .
\end{aligned}
$$

Now we estimate $\left\|\Pi_{h} \boldsymbol{\sigma}-\widetilde{\boldsymbol{\sigma}}_{h}\right\|$,

$$
\left(\boldsymbol{\sigma}-\widetilde{\boldsymbol{\sigma}}_{h}, \Pi_{h} \boldsymbol{\sigma}-\widetilde{\boldsymbol{\sigma}}_{h}\right)=\left(a\left(\boldsymbol{\lambda}-\tilde{\boldsymbol{\lambda}}_{h}\right), \Pi_{h} \boldsymbol{\sigma}-\widetilde{\boldsymbol{\sigma}}_{h}\right) .
$$


It is easy to see that

$$
\begin{aligned}
&\left\|\Pi_{h} \boldsymbol{\sigma}-\widetilde{\boldsymbol{\sigma}}_{h}\right\|^{2} \\
&=\left(\Pi_{h} \boldsymbol{\sigma}-\boldsymbol{\sigma}, \Pi_{h} \boldsymbol{\sigma}-\tilde{\boldsymbol{\sigma}}_{h}\right)+\left(a\left(\boldsymbol{\lambda}-\tilde{\boldsymbol{\lambda}}_{h}\right), \Pi_{h} \boldsymbol{\sigma}-\tilde{\boldsymbol{\sigma}}_{h}\right) \\
&=\left(\Pi_{h} \boldsymbol{\sigma}-\boldsymbol{\sigma}, \Pi_{h} \boldsymbol{\sigma}-\tilde{\boldsymbol{\sigma}}_{h}\right)+\left(a\left(\boldsymbol{\lambda}-R_{h} \boldsymbol{\lambda}\right), \Pi_{h} \boldsymbol{\sigma}-\tilde{\boldsymbol{\sigma}}_{h}\right) \\
& \quad+\left(a\left(R_{h} \boldsymbol{\lambda}-\tilde{\boldsymbol{\lambda}}_{h}\right), \Pi_{h} \boldsymbol{\sigma}-\tilde{\boldsymbol{\sigma}}_{h}\right) \\
& \leq C\left(\left\|\Pi_{h} \boldsymbol{\sigma}-\boldsymbol{\sigma}\right\|+\left\|\boldsymbol{\lambda}-R_{h} \lambda\right\|+\left\|u_{1}\right\|+\left\|u_{2}\right\|\right) \\
& \times\left\|\Pi_{h} \boldsymbol{\sigma}-\tilde{\boldsymbol{\sigma}}_{h}\right\| .
\end{aligned}
$$

By the properties of projection $\Pi_{h}$, we get

$$
\begin{array}{r}
\left\|\Pi_{h} \boldsymbol{\sigma}-\tilde{\boldsymbol{\sigma}}_{h}\right\| \leq C h^{r}\left(\|u\|_{r}+\|\lambda\|_{r}+\|\boldsymbol{\sigma}\|_{r}+\left\|u_{1}\right\|\right), \\
0 \leq r \leq k+1,
\end{array}
$$

which proves (49) and (53).

Now we estimate $\left\|R_{h} \lambda-\tilde{\lambda}_{h}\right\|$,

$$
\begin{aligned}
&\left(a\left(R_{h} \boldsymbol{\lambda}-\tilde{\boldsymbol{\lambda}}_{h}\right), R_{h} \boldsymbol{\lambda}-\tilde{\boldsymbol{\lambda}}_{h}\right) \\
&=\left(a\left(\boldsymbol{\lambda}-\tilde{\boldsymbol{\lambda}}_{h}, R_{h} \boldsymbol{\lambda}-\tilde{\boldsymbol{\lambda}}_{h}\right)\right. \\
&+\left(a\left(R_{h} \boldsymbol{\lambda}-\boldsymbol{\lambda}\right), R_{h} \boldsymbol{\lambda}-\tilde{\boldsymbol{\lambda}}_{h}\right) \\
&=\left(\boldsymbol{\sigma}-\Pi_{h} \boldsymbol{\sigma}, R_{h} \boldsymbol{\lambda}-\tilde{\boldsymbol{\lambda}}_{h}\right)+\left(\Pi_{h} \boldsymbol{\sigma}-\tilde{\boldsymbol{\sigma}}_{h}, R_{h} \boldsymbol{\lambda}-\tilde{\boldsymbol{\lambda}}_{h}\right) \\
&+\left(a\left(R_{h} \boldsymbol{\lambda}-\boldsymbol{\lambda}\right), R_{h} \boldsymbol{\lambda}-\tilde{\boldsymbol{\lambda}}_{h}\right) \\
&=\left(\boldsymbol{\sigma}-\Pi_{h} \boldsymbol{\sigma}, R_{h} \boldsymbol{\lambda}-\tilde{\boldsymbol{\lambda}}_{h}\right)+\left(\mathbf{c}\left(u-\tilde{u}_{h}\right), \Pi_{h} \boldsymbol{\sigma}-\tilde{\boldsymbol{\sigma}}_{h}\right) \\
&+\left(a\left(R_{h} \boldsymbol{\lambda}-\boldsymbol{\lambda}\right), R_{h} \boldsymbol{\lambda}-\tilde{\boldsymbol{\lambda}}_{h}\right) \\
& \leq\left(\left\|\boldsymbol{\sigma}-\Pi_{h} \boldsymbol{\sigma}\right\|+C\left\|\boldsymbol{\lambda}-R_{h} \lambda\right\|\right)\left\|R_{h} \boldsymbol{\lambda}-\tilde{\boldsymbol{\lambda}}_{h}\right\| \\
&+C\left\|u_{1}+u_{2}\right\|\left\|\Pi_{h} \boldsymbol{\sigma}-\tilde{\boldsymbol{\sigma}}_{h}\right\| \\
& \leq C\left(\left\|\boldsymbol{\sigma}-\Pi_{h} \boldsymbol{\sigma}\right\|^{2}+\left\|u_{1}\right\|^{2}+\left\|u_{2}\right\|^{2}+\left\|R_{h} \boldsymbol{\lambda}-\lambda\right\|^{2}\right) \\
&+\frac{c_{0}}{2}\left\|R_{h} \boldsymbol{\lambda}-\tilde{\boldsymbol{\lambda}}_{h}\right\|^{2},
\end{aligned}
$$

where

$$
\left(a\left(R_{h} \lambda-\tilde{\lambda}_{h}\right), R_{h} \lambda-\tilde{\lambda}_{h}\right) \geq c_{0}\left\|R_{h} \lambda-\tilde{\lambda}_{h}\right\|^{2} .
$$

From (66) and (67), we know

$$
\begin{array}{r}
\left\|R_{h} \lambda-\tilde{\lambda}_{h}\right\| \leq C\left(h^{r}\left(\|u\|_{r}+\|\lambda\|_{r}+\|\boldsymbol{\sigma}\|_{r}\right)+\left\|u_{1}\right\|\right), \\
\left\|\lambda-\tilde{\lambda}_{h}\right\| \leq C\left(h^{r}\left(\|u\|_{r}+\|\lambda\|_{r}+\|\boldsymbol{\sigma}\|_{r}\right)+\left\|u_{1}\right\|\right), \\
0 \leq r \leq k+1 .
\end{array}
$$

By Lemma 2 , if $k=0$, we have that

$$
\begin{aligned}
&\left\|\lambda-\tilde{\lambda}_{h}\right\|=\left\|\lambda_{1}\right\| \\
& \leq C h\left(\|u\|_{1}+\|\lambda\|_{1}+\|\sigma\|_{1}\right. \\
&\left.\quad+\int_{0}^{t}\left(\|u\|_{1}+\|\lambda\|_{1}+\|\boldsymbol{\sigma}\|_{1}\right) d \tau\right) .
\end{aligned}
$$

If $k \geq 1,2 \leq r \leq k+1$, we have that

$$
\begin{aligned}
\left\|\lambda-\tilde{\lambda}_{h}\right\| \leq C h^{r}\left(\|u\|_{r}+\|\lambda\|_{r}+\|\boldsymbol{\sigma}\|_{r}\right. \\
\left.\quad+\int_{0}^{t}\left(\|u\|_{r-1}+\|\lambda\|_{r-1}\|\boldsymbol{\sigma}\|_{r-1}\right) d t\right) .
\end{aligned}
$$

Using the estimate of $\left\|\Pi_{h} \boldsymbol{\sigma}-\tilde{\boldsymbol{\sigma}}_{h}\right\|$, we obtain

$$
\begin{array}{r}
\left\|\boldsymbol{\sigma}-\tilde{\boldsymbol{\sigma}}_{h}\right\| \leq C\left(h^{r}\left(\|u\|_{r}+\|\lambda\|_{r}+\|\boldsymbol{\sigma}\|_{r}\right)+\left\|u_{1}\right\|\right), \\
0 \leq r \leq k+1 .
\end{array}
$$

If $k=0$, we get

$$
\begin{aligned}
&\left\|\boldsymbol{\sigma}-\tilde{\boldsymbol{\sigma}}_{h}\right\| \\
& \leq C h\left(\|u\|_{1}+\|\lambda\|_{1}+\|\boldsymbol{\sigma}\|_{1}\right. \\
&\left.\quad+\int_{0}^{t}\left(\|u\|_{1}+\|\lambda\|_{1}+\|\boldsymbol{\sigma}\|_{1}\right) d t\right) .
\end{aligned}
$$

If $k \geq 1,2 \leq r \leq k+1$, we get

$$
\begin{aligned}
& \left\|\boldsymbol{\sigma}-\tilde{\boldsymbol{\sigma}}_{h}\right\| \\
& \leq C^{r}\left(\|u\|_{r}+\|\lambda\|_{r}+\|\boldsymbol{\sigma}\|_{r}\right. \\
& \left.\quad+\int_{0}^{t}\left(\|u\|_{r-1}+\|\lambda\|_{r-1}+\|\boldsymbol{\sigma}\|_{r-1}\right) d t\right) .
\end{aligned}
$$

(III) By Lemma 2, we have that

$$
\left\|p_{h} u-\widetilde{u}_{h}\right\|=\left\|u_{1}\right\| \leq C h^{r+1-\delta_{k 0}}
$$

$$
\times \int_{0}^{t}\left(\|u\|_{r}+\|\lambda\|_{r}+\|\boldsymbol{\sigma}\|_{r}\right) d \tau, \quad 0 \leq r \leq k+1,
$$

$\left\|p_{h} u_{t}-\tilde{u}_{h, t}\right\|$

$$
\begin{array}{r}
\leq C h^{r+1-\delta_{k 0}}\left(\|u\|_{r}+\|\lambda\|_{r}+\|\boldsymbol{\sigma}\|_{r}\right. \\
\left.+\int_{0}^{t}\left(\|u\|_{r}+\|\lambda\|_{r}+\|\boldsymbol{\sigma}\|_{r}\right) d t\right), \\
0 \leq r \leq k+1 .
\end{array}
$$


If $k=0$, we know

$$
\begin{aligned}
&\left\|u-\tilde{u}_{h}\right\| \leq\left\|u-p_{h} u\right\|+\left\|u_{1}\right\| \leq C h\left(\|u\|_{1}+\int_{0}^{t}\left(\|u\|_{1}+\|\lambda\|_{1}+\|\boldsymbol{\sigma}\|_{1}\right) d t\right) \\
&\left\|u_{t}-\tilde{u}_{h, t}\right\| \\
& \leq C h\left(\left\|u_{t}\right\|_{1}+\|u\|_{1}+\|\lambda\|_{1}+\|\boldsymbol{\sigma}\|_{1}\right. \\
&\left.+\int_{0}^{t}\left(\|u\|_{1}+\|\lambda\|_{1}+\|\boldsymbol{\sigma}\|_{1}\right) d t\right) .
\end{aligned}
$$

If $k \geq 1,2 \leq r \leq k+1$, we know

$$
\begin{aligned}
& \left\|u-\tilde{u}_{h}\right\| \\
& \leq C h^{r}\left(\|u\|_{r}+\int_{0}^{t}\left(\|u\|_{r-1}+\|\lambda\|_{r-1}+\|\sigma\|_{r-1}\right) d t\right), \\
& \left\|u_{t}-\tilde{u}_{h, t}\right\| \\
& \leq C h^{r}\left(\left\|u_{t}\right\|_{r}+\|u\|_{r-1}+\|\lambda\|_{r-1}\right. \\
& \left.\quad+\int_{0}^{t}\left(\|u\|_{r-1}+\|\lambda\|_{r-1}+\|\sigma\|_{r-1}\right) d t\right) .
\end{aligned}
$$

The proof is completed.

Remark 4. The estimate results of (46)-(53) are optimal. If $k \geq 1$, the estimate results of (43)-(45) are superconvergent.

\section{Main Result}

In this section, we consider error estimates for the continuous-in-time mixed finite element approximation. Define

$$
\begin{gathered}
u-u_{h}=\left(u-\tilde{u}_{h}\right)+\left(\tilde{u}_{h}-u_{h}\right)=u_{3}+u_{4}, \\
\lambda-\lambda_{h}=\left(\lambda-\tilde{\lambda}_{h}\right)+\left(\tilde{\lambda}_{h}-\lambda_{h}\right)=\lambda_{1}+\lambda_{2}, \\
\sigma-\sigma_{h}=\left(\sigma-\widetilde{\sigma}_{h}\right)+\left(\widetilde{\boldsymbol{\sigma}}_{h}-\sigma_{h}\right)=\sigma_{1}+\sigma_{2}, \\
\left\|u, u_{t}, \lambda, \boldsymbol{\sigma}\right\|_{r}=\|u\|_{r}+\left\|u_{t}\right\|_{r}+\|\lambda\|_{r}+\|\boldsymbol{\sigma}\|_{r}, \\
\mid\left\|u, u_{t}, \lambda, \boldsymbol{\sigma}\right\|_{r}=\int_{0}^{t}\left(\|u\|_{r}+\left\|u_{t}\right\|_{r}+\|\lambda\|_{r}+\|\boldsymbol{\sigma}\|_{r}\right) d t .
\end{gathered}
$$

Theorem 5. Let $(u, \lambda, \sigma)$ and $\left(u_{h}, \lambda_{h}, \sigma_{h}\right)$ be the solution of (9) and (11), respectively. If $u, \lambda$, and $\sigma$ are sufficiently smooth, then there exist positive constants $C$ such that
(I) if $k=0$, then

$$
\begin{gathered}
\left\|u-u_{h}\right\| \leq C h\left(\|u\|_{1}+\left\|u, u_{t}, \lambda, \boldsymbol{\sigma}\right\|_{1}\right), \\
\left\|u_{t}-u_{h, t}\right\| \leq C h\left(\left\|u, u_{t}, \lambda, \boldsymbol{\sigma}\right\|_{1}+|\|u, \lambda, \boldsymbol{\sigma}\||_{1}\right), \\
\left\|\lambda-\lambda_{h}\right\| \leq C h\left(\left\|u, u_{t}, \lambda, \boldsymbol{\sigma}\right\|_{1}+\left\|u, u_{t}, \lambda, \boldsymbol{\sigma}\right\|_{1}\right), \\
\left\|\boldsymbol{\sigma}-\boldsymbol{\sigma}_{h}\right\| \leq C h\left(\left\|u, u_{t}, \lambda, \boldsymbol{\sigma}\right\|_{1}+\left\|u, u_{t}, \lambda, \boldsymbol{\sigma}\right\|_{1}\right), \\
\left\|\operatorname{div}\left(\boldsymbol{\sigma}-\boldsymbol{\sigma}_{h}\right)\right\| \\
\leq C h\left(\|u\|_{1}+\left\|u_{t}\right\|_{1}+\|\boldsymbol{\sigma}\|_{2}+\left\|u, u_{t}, \lambda, \boldsymbol{\sigma}\right\|_{1}\right),
\end{gathered}
$$

(II) if $k \geq 1$ and $2 \leq r \leq k+1$, then

$$
\left\|u-u_{h}\right\| \leq C h^{r}\left(\|u\|_{r}+\mid\left\|u, u_{t}, \lambda, \sigma\right\|_{r-1}\right),
$$

$\left\|u_{t}-u_{h, t}\right\|$

$$
\leq C h^{r}\left(\left\|u_{t}\right\|_{r}+\|u\|_{r-1}+\|\lambda\|_{r-1}+\|\sigma\|_{r-1}\right.
$$

$$
\left.+\int_{0}^{t}\left(\|u\|_{r-1}+\left\|u_{t}\right\|_{r}+\|\lambda\|_{r-1}+\|\boldsymbol{\sigma}\|_{r-1}\right) d t\right),
$$

$\left\|\lambda-\lambda_{h}\right\|$

$\leq C h^{r}\left(\|u, \lambda, \sigma\|_{r}\right.$

$$
\left.+\int_{0}^{t}\left(\left\|u_{t}\right\|_{r}+\|u\|_{r-1}+\|\lambda\|_{r-1}+\|\boldsymbol{\sigma}\|_{r-1}\right) d t\right),
$$

$\left\|\sigma-\sigma_{h}\right\|$

$$
\begin{aligned}
\leq \operatorname{Ch}^{r} & \left(\|u, \lambda, \boldsymbol{\sigma}\|_{r}\right. \\
& \left.+\int_{0}^{t}\left(\left\|u_{t}\right\|_{r}+\|u\|_{r-1}+\|\lambda\|_{r-1}+\|\boldsymbol{\sigma}\|_{r-1}\right) d t\right),
\end{aligned}
$$

$$
\begin{aligned}
& \left\|\operatorname{div}\left(\boldsymbol{\sigma}-\boldsymbol{\sigma}_{h}\right)\right\| \\
& \leq \operatorname{Ch}^{r}\left(\left\|u_{t}\right\|_{r}+\|\lambda\|_{r}+\|\boldsymbol{\sigma}\|_{r+1}+\|u\|_{r-1}\right. \\
& \left.\quad+\int_{0}^{t}\left(\left\|u_{t}\right\|_{r}+\|u\|_{r-1}+\|\lambda\|_{r-1}+\|\boldsymbol{\sigma}\|_{r-1}\right) d t\right) .
\end{aligned}
$$

Proof. From (9) and (19), we have the following error equation:

$$
\begin{array}{r}
\left(u_{4, t}+u_{3, t}, w\right)+\left(\operatorname{div} \sigma_{2}, w\right)=0, \\
\forall w \in W_{h}, 0<t \leq T,
\end{array}
$$




$$
\begin{gathered}
\left(\boldsymbol{\sigma}_{2}, \boldsymbol{\tau}\right)-\left(a \lambda_{2}, \boldsymbol{\tau}\right)=0, \\
\forall \boldsymbol{\tau} \in \Lambda_{h}, 0<t \leq T, \\
\left(\lambda_{2}, \mathbf{v}\right)-\left(u_{4, t}+b u_{4}, \operatorname{div} \mathbf{v}\right)+\left(\mathbf{c} u_{4}, \mathbf{v}\right)=0, \\
\forall \mathbf{v} \in \mathbf{V}_{h}, 0<t \leq T, \\
u_{4}(0)=0 .
\end{gathered}
$$

In (85), choosing $\omega=u_{4, t}+p_{h}\left(b u_{4}\right), \boldsymbol{\tau}=\lambda_{2}$, and $\mathbf{v}=\sigma_{2}$, we have

$$
\begin{aligned}
\left(\lambda_{2}, \sigma_{2}\right) & +\left(u_{4, t}, u_{4, t}\right) \\
= & -\left(u_{3, t}, u_{4, t}+p_{h}\left(b u_{4}\right)\right)-\left(u_{4, t}, p_{h}\left(b u_{4}\right)\right) \\
& -\left(\mathbf{c} u_{4}, \sigma_{2}\right) .
\end{aligned}
$$

In the second equation of (85), letting $\boldsymbol{\tau}=\boldsymbol{\sigma}_{2}$, we know

$$
\sigma_{2} \leq C \lambda_{2} \text {. }
$$

Further, using $\varepsilon$-inequality to (86), we get

$$
\left\|u_{4, t}\right\|^{2}+\left\|\lambda_{2}\right\|^{2} \leq C\left(\left\|u_{4}\right\|^{2}+\left\|u_{3, t}\right\|^{2}\right) .
$$

Hence,

$$
\begin{aligned}
\left\|u_{4}\right\| & =\left\|\int_{0}^{t} u_{4, t} d \tau\right\| \\
& \leq C \int_{0}^{t}\left\|u_{4, t}\right\| d \tau \leq C \int_{0}^{t}\left(\left\|u_{4}\right\|+\left\|u_{3, t}\right\|\right) d t .
\end{aligned}
$$

Using Gronwall's inequality to (89), we obtain

$$
\left\|u_{4}\right\| \leq C \int_{0}^{t}\left\|u_{3, t}\right\| d t
$$

Further, we know

$$
\left\|\sigma_{2}\right\|+\left\|u_{4, t}\right\|+\left\|\lambda_{2}\right\| \leq C\left(\left\|u_{3, t}\right\|+\int_{0}^{t}\left\|u_{3, t}\right\| d t\right) .
$$

Noting the first equation of (78), we get

$$
\left\|\operatorname{div} \sigma_{2}\right\| \leq\left\|u_{3, t}\right\|+\left\|u_{4, t}\right\| \leq C\left(\left\|u_{3, t}\right\|+\int_{0}^{t}\left\|u_{3, t}\right\| d t\right) .
$$

So we have that

$$
\begin{gathered}
\left\|u-u_{h}\right\| \leq\left\|u_{3}\right\|+C \int_{0}^{t}\left\|u_{3, t}\right\| d t, \\
\left\|u_{t}-u_{h, t}\right\| \leq C\left(\left\|u_{3, t}\right\|+\int_{0}^{t}\left\|u_{3, t}\right\| d t\right), \\
\left\|\lambda-\lambda_{h}\right\| \leq\left\|\lambda_{1}\right\|+C\left(\left\|u_{3, t}\right\|+\int_{0}^{t}\left\|u_{3, t}\right\| d t\right), \\
\left\|\boldsymbol{\sigma}-\boldsymbol{\sigma}_{h}\right\| \leq\left\|\boldsymbol{\sigma}_{1}\right\|+C\left(\left\|u_{3, t}\right\|+\int_{0}^{t}\left\|u_{3, t}\right\| d t\right), \\
\left\|\operatorname{div}\left(\boldsymbol{\sigma}-\boldsymbol{\sigma}_{h}\right)\right\| \leq\left\|\operatorname{div} \boldsymbol{\sigma}_{1}\right\|+\left\|\operatorname{div} \boldsymbol{\sigma}_{2}\right\| .
\end{gathered}
$$

Together with the results of Lemmas 2 and 3, the proof is completed.
Remark 6. The estimate results of (78)-(84) are optimal.

\section{Acknowledgments}

This work is supported by the National Natural Science Foundation of China (nos. 11171190, 11101246, and 11101247) and the Natural Science Foundation of Shandong Province (ZR2011AQ020). The authors thank the anonymous referees for their constructive comments and suggestions, which led to improvements in the presentation.

\section{References}

[1] N. Li, F. Gao, and T. Zhang, "An expanded mixed finite element method for Sobolev equation," Journal of Computational Analysis and Applications, vol. 15, no. 3, pp. 535-543, 2013.

[2] T. Sun, "A Godunov-mixed finite element method on changing meshes for the nonlinear Sobolev equations," Abstract and Applied Analysis, vol. 2012, Article ID 413718, 19 pages, 2012.

[3] R. E. Ewing, "Numerical solution of Sobolev partial differential equations," SIAM Journal on Numerical Analysis, vol. 12, pp. 345-363, 1975.

[4] D. Shi and Y. Zhang, "High accuracy analysis of a new nonconforming mixed finite element scheme for Sobolev equations," Applied Mathematics and Computation, vol. 218, no. 7, pp. 31763186, 2011.

[5] P. G. Ciarlet, The Finite Element Method for Elliptic Equations, North-Holland, Amsterdam, The Netherlands, 1978.

[6] I. Babuska, "Error-bounds for finite element method," Numerische Mathematik, vol. 16, no. 4, pp. 322-333, 1970.

[7] F. Brezzi, "On the existence, uniqueness and approximation of saddle-point problems arising from Lagrangian multipliers," RAIRO Mathematical Modelling and Numerical Analysis, vol. 8, no. 2, pp. 129-151, 1974.

[8] R. S. Falk and J. E. Osborn, "Error estimates for mixed methods," RAIRO Analyse Numérique, vol. 14, no. 3, pp. 249-277, 1980.

[9] F. Brezzi and M. Fortin, Mixed and Hybrid Finite Element Methods, vol. 15 of Springer Series in Computational Mathematics, Springer, Berlin, Germany, 1991.

[10] J. E. Roberts and J. M. Thomas, "Mixed and hybrid methods," in Handbook of Numerical Analysis, vol. 2, pp. 523-639, NorthHolland, Amsterdam, The Netherlands, 1991.

[11] Z. Chen, "Expanded mixed finite element methods for linear second-order elliptic problems. I," RAIRO Mathematical Modelling and Numerical Analysis, vol. 32, no. 4, pp. 479-499, 1998.

[12] Z. Chen, "Expandedmixed finite elementmethods for quasilinear second-order elliptic problems," RAIROMathematical Modelling and Numerical Analysis, vol. 32, no. 4, pp. 500-520, 1998.

[13] L. Guo and H. Z. Chen, "An expanded characteristic-mixed finite element method for a convection-dominated transport problem," Journal of Computational Mathematics, vol. 23, no. 5, pp. 479-490, 2005.

[14] W. Liu, H. X. Rui, and H. Guo, "A two-grid method with expanded mixed element for nonlinear reaction-diffusion equations," Acta Mathematicae Applicatae Sinica (English Series), vol. 27, no. 3, pp. 495-502, 2011.

[15] H. Che, Z. Zhou, and Z. Jiang, "H1-Galerkin expanded mixed finite element methods for nonlinear pseudo-parabolic integrodifferential equations," Numerical Methods for Partial Differential Equations, vol. 29, no. 3, pp. 799-817, 2013. 
[16] R. A. Adams, Sobolev Spaces, vol. 65, Academic Press, New York, NY, USA, 1975.

[17] P. A. Raviart and J. M. Thomas, "A mixed finite element method for 2nd order elliptic problems," in Mathematical Aspects of Finite Element Methods, vol. 606 of Lecture Notes in Mathematics, pp. 292-315, Springer, Berlin, Germany, 1977.

[18] F. Brezzi, J. Douglas Jr., and L. D. Marini, "Two families of mixed finite elements for second order elliptic problems," Numerische Mathematik, vol. 47, no. 2, pp. 217-235, 1985.

[19] F. Brezzi, J. Douglas Jr., M. Fortin, and L. D. Marini, "Efficient rectangular mixed finite elements in two and three space variables," RAIRO Mathematical Modelling and Numerical Analysis, vol. 21, no. 4, pp. 581-604, 1987. 


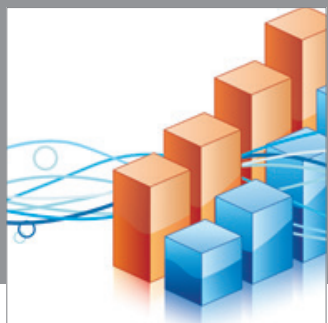

Advances in

Operations Research

mansans

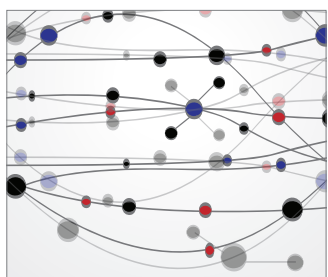

The Scientific World Journal
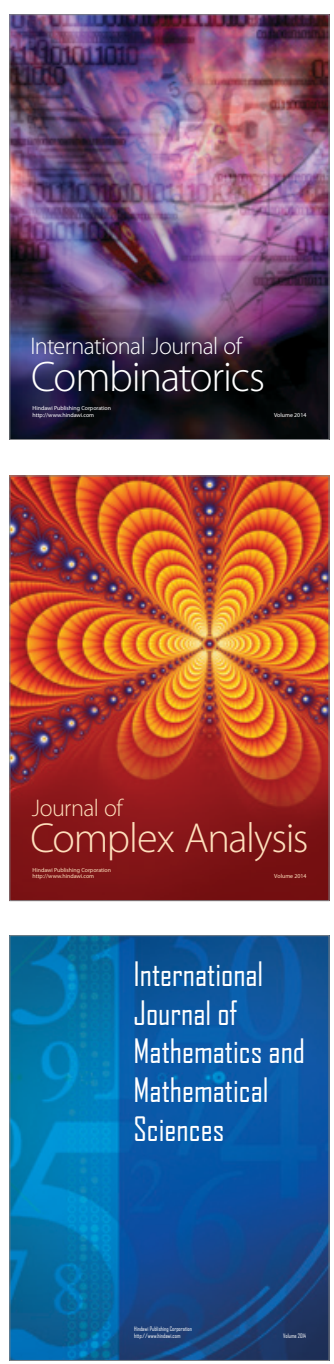
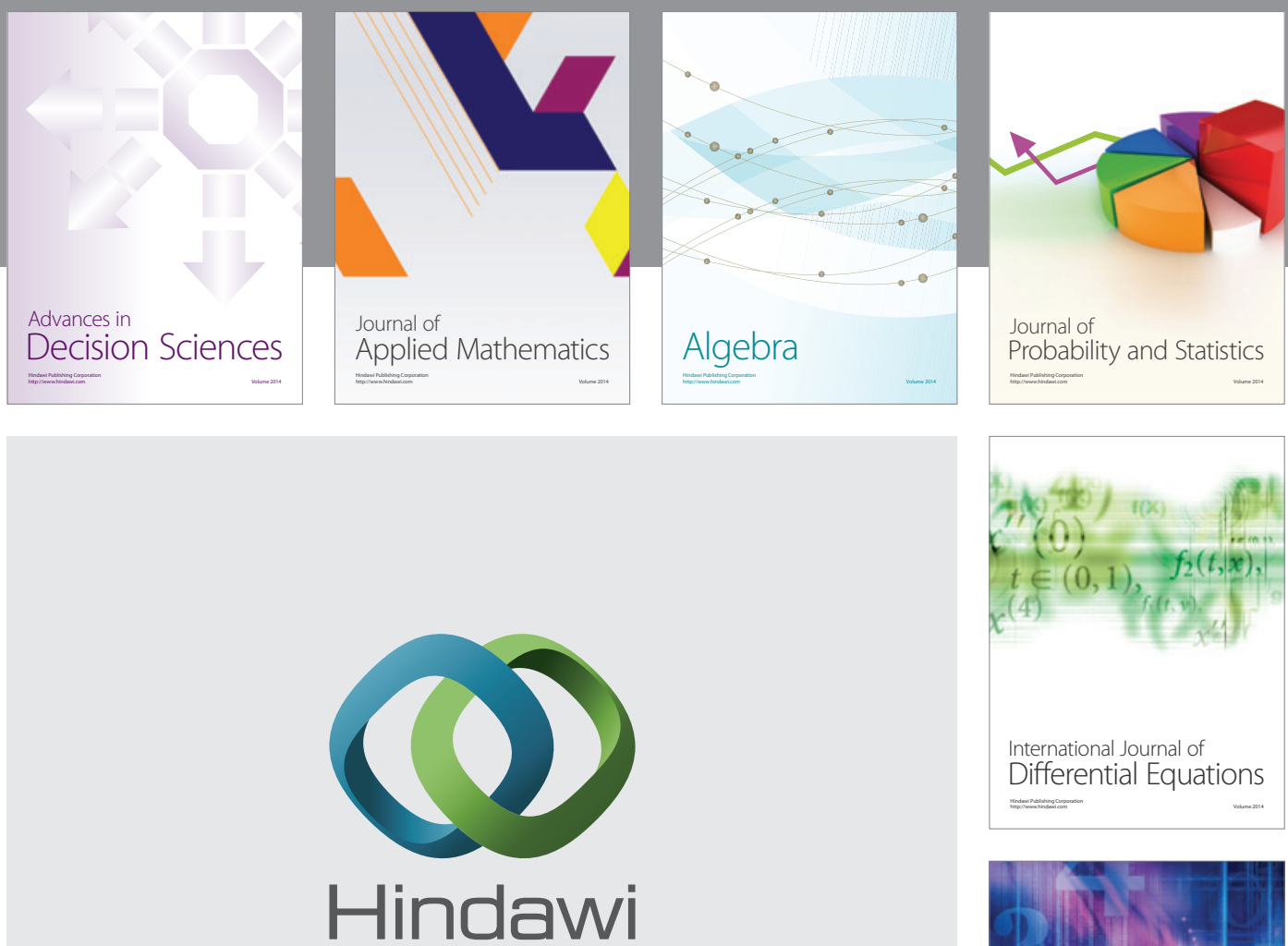

Submit your manuscripts at http://www.hindawi.com
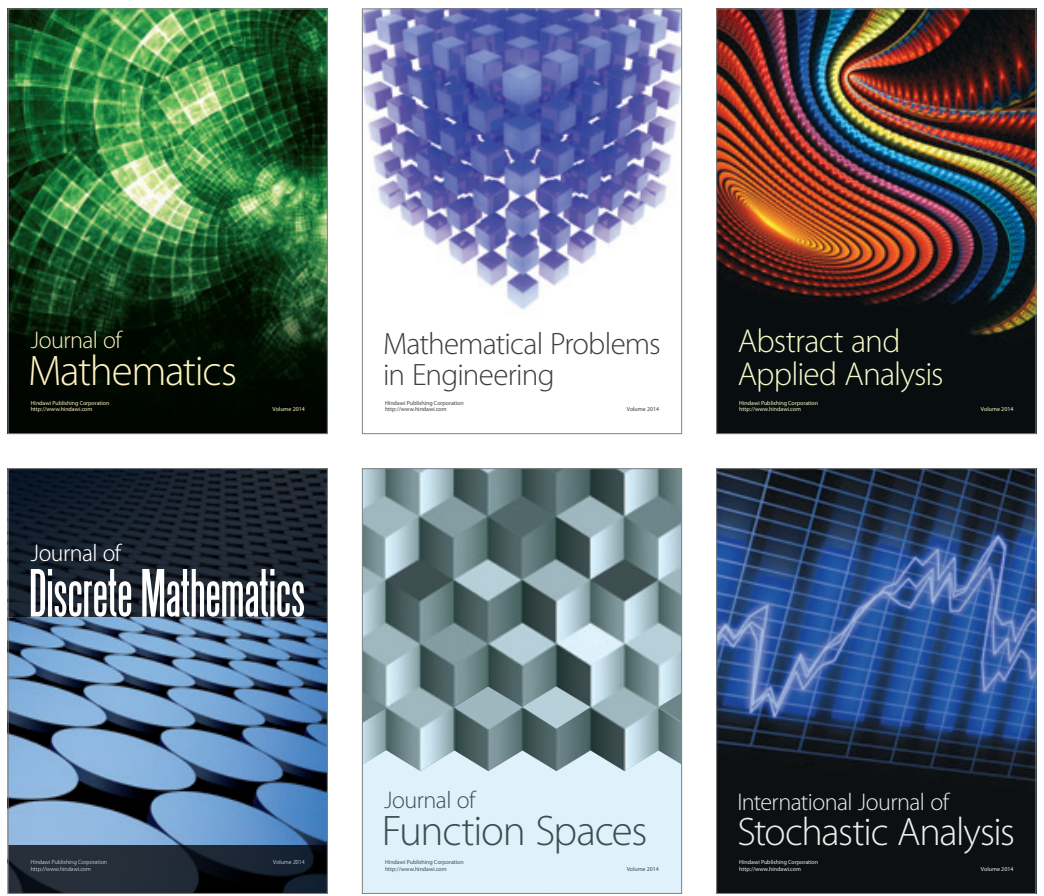

Journal of

Function Spaces

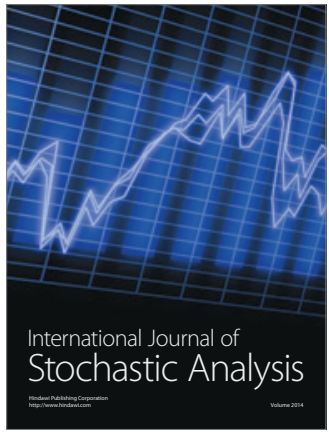

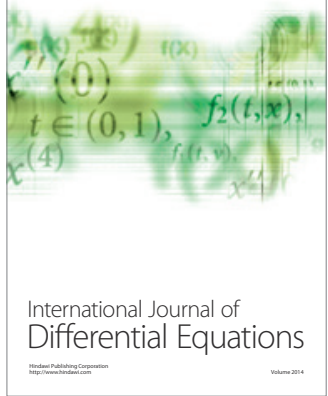
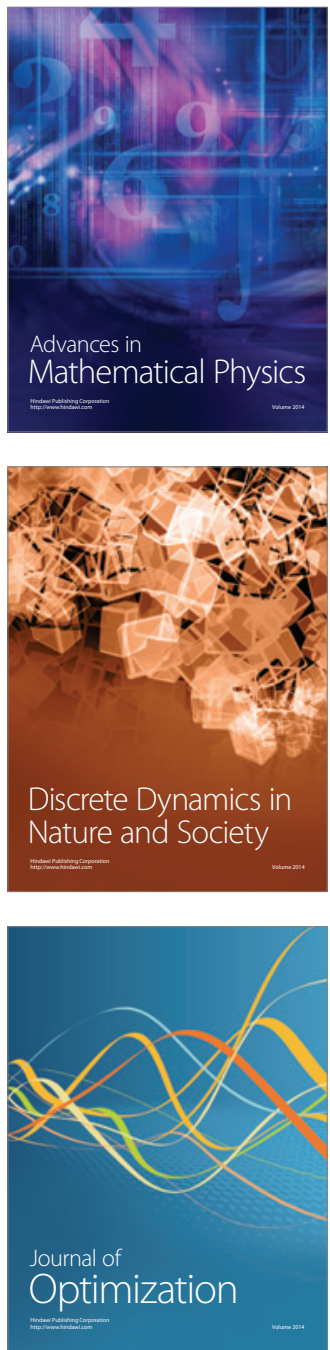